\title{
Extensive Bowel Ischemia with Heavy Alcohol Consumption: Report of a Case
}

\author{
Ji Hyoun Lee, Gyoung Tae Noh ${ }^{1}$, Ryung-Ah Lee ${ }^{1}$ \\ Department of Surgery, Gyeonggi-do Medical Center Pocheon Hospital, Pocheon; ${ }^{1}$ Department of Surgery, Ewha Womans University \\ Mokdong Hospital, Ewha Womans University School of Medicine, Seoul, Korea
}

\begin{abstract}
Alcohol is well-recognized systemic toxin that causes numerous adverse effects, including psychosocial problems, fatal myocardial infarction, stroke and atherosclerosis. The intra-abdominal complications caused by acute alcohol consumption have not been defined. We report an 80-year-old man with sub-acute small bowel and colonic ischemia after heavy alcohol intake in one sitting. We performed a resection of gangrenous bowel segments. Microscopically, there were diffuse infarction, with vasoconstriction of the mesenteric vessels in the ileum and colon without any thrombotic occlusion of the intestinal vessels. The clinicians should always be assured by confirmation of a history of recent substance abuse in patients with unexplained abdominal pain, and mesenteric ischemia should be considered in the differential diagnosis of acute or chronic abdominal pain in consumers of alcohol.
\end{abstract}

Keywords: Alcohols; Intestinal ischemia; Vasoconstriction

\section{INTRODUCTION}

Acute mesenteric ischemia (AMI) is an emergency situation that is caused by circulatory disturbances of mesenteric vessels and is frequently associated with atherosclerosis and cardiovascular disorders. AMI is a complex disease entity including acute mesenteric arterial embolism and thrombosis, mesenteric venous thrombosis, and non-occlusive mesenteric ischemia (NOMI). NOMI results from low-velocity blood flow states or from mesenteric vasoconstrictions caused by some drugs.

Intestinal ischemia is a relatively uncommon cause of abdominal pain. The abdominal pain caused by intestinal ischemia is not proportional to the physical examination findings, especially in

Received: May 31, 2011 • Revised: November 3, 2011

Accepted: November 21, 2011

Correspondence to: Ryung-Ah Lee, M.D.

Department of Surgery, Ewha Womans University Mokdong Hospital, Ewha Womans University School of Medicine, 1071 Anyangcheon-ro, Yangcheon-gu, Seoul 158-710, Korea

Tel: +82-2-2650-2659, Fax: +82-2-2644-7984

E-mail: ralee@ewha.ac.kr

(C) 2012 The Korean Society of Coloproctology

This is an open-access article distributed under the terms of the Creative Commons Attribution NonCommercial License (http://creativecommons.org/licenses/by-nc/3.0) which permits unrestricted noncommercial use, distribution, and reproduction in any medium, provided the original work is properly cited. elderly persons. Although computed tomography (CT), magnetic resonance imaging and angiography are valuable diagnostic steps, definite diagnosis of intestinal ischemia frequently depends upon the clinical history because the representation would be subtle and obscure. The majority of patients with ischemic colitis can be treated medically, but approximately $20 \%$ of the patients clinically deteriorate to develop peritonitis and sepsis, necessitating surgical intervention with an associated $60 \%$ mortality rate $[1,2]$. A laparotomy is helpful for rapid confirmation of the diagnosis and for optimal treatment, which often consists of revascularization and resection of the affected bowel.

Alcohol is well recognized as a systemic toxin that causes serious health problems, including psychosocial problems, myocardial infarction, stroke and atherosclerosis. Alcohol-related complications are, therefore, of clinical importance. However, intestinal complications caused by alcohol consumption are not well documented yet. We reported a case of sub-acute ileal and colonic ischemia in an elderly patient, not having a history of cardiovascular disorders, after heavy alcohol intake, and we present a review of the literature.

\section{CASE REPORT}

An 80-year-old male patient was admitted due to uncontrolled diarrhea and abdominal pain accompanied by fever. He became 
Journal of The Korean Society of Extensive Bowel Ischemia with Heavy Alcohol Consumption: Report of a Case

Coloproctology Ji Hyoun Lee, et al.

unconscious after drinking 1,800 $\mathrm{mL}$ of Soju (Korean traditional drink, 25\% distilled alcohol) in one sitting and was then admitted to a local clinic. After conservative management, he woke up coherent, but he was referred to Ewha Womans University Mokdong Hospital because his diarrhea, fever and abdominal pain had not subsided after 10 days. He had no history of chronic diseases such as diabetes, cardiovascular disease, hypertension, hepatitis or smoking. He had had a drinking habit of half a bottle of Soju $(90 \mathrm{~mL})$ daily for forty years.

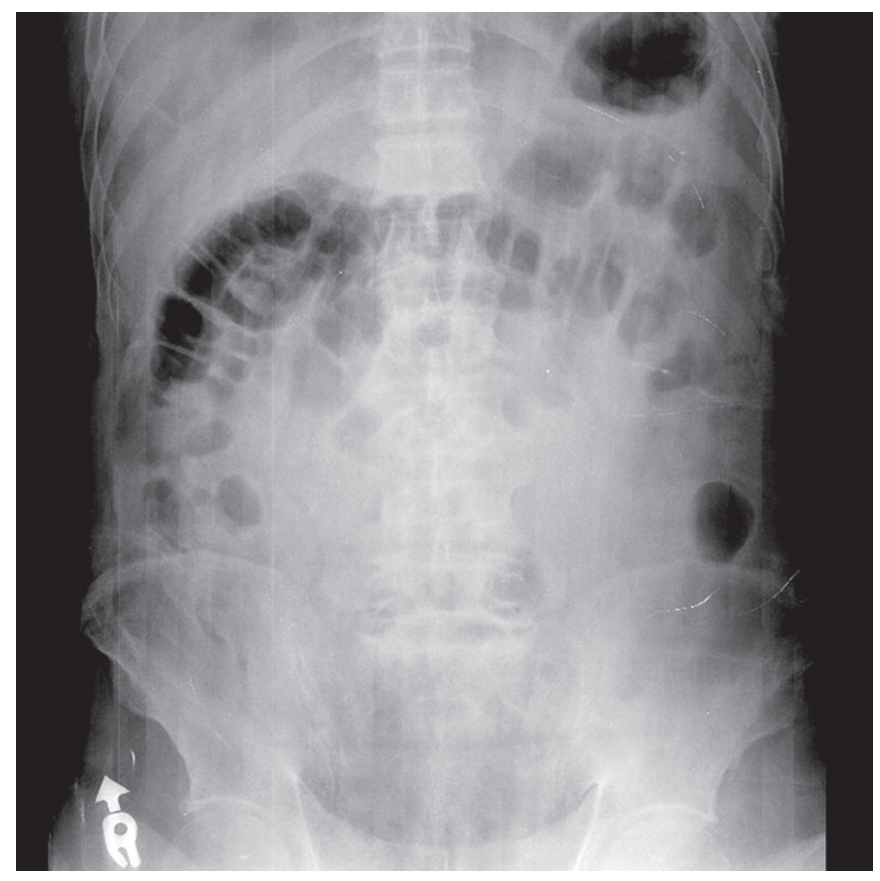

Fig. 1. Upright and supine simple abdomen film on admission day. It is noted that moderate ileus features were found around the small bowel.
On admission, his blood pressure was 110/60 $\mathrm{mmHg}$, his pulse rate was 84 beats/min, his respiratory rate was 24 breaths $/ \mathrm{min}$, his body temperature was $36^{\circ} \mathrm{C}$, and his mental status was clear. $\mathrm{He}$ had continuously suffered from diarrhea from the onset of the illness, 6 to 7 times daily, including night-time defecation. Upon physical examination, there was mild generalized tenderness with abdominal distension on the whole abdomen and hyperactive bowel sound. Laboratory data included the following: leukocyte count, $14,100 / \mu \mathrm{L}$ with no leftward shift (neutrophil count, $61.8 \%$ ); hemoglobin level, $12.1 \mathrm{~g} / \mathrm{dL}$; platelet count, 263,000/dL; total protein, $4.8 \mathrm{~g} / \mathrm{dL}$; albumin, $2.2 \mathrm{~g} / \mathrm{dL}$; aspartate transaminase, $78 \mathrm{IU} / \mathrm{L}$; alanine transaminase, $103 \mathrm{IU} / \mathrm{L}$; blood urea nitrogen $8 \mathrm{mg} / \mathrm{dL}$; serum creatinine, $0.7 \mathrm{mg} / \mathrm{dL}$; amylase, $82 \mathrm{U} / \mathrm{L}$; creatine kinase, $48 \mathrm{IU} / \mathrm{L}$; and lactate dehydrogenase, $396 \mathrm{IU} / \mathrm{L}$. Upright and supine roentgenograms of the abdomen showed the paralytic ileus with bowel distension (Fig. 1). Abdominal CT scan showed circumferential wall thickening of the bowel from the distal ileum to the ascending colon (Fig. 2). Considering the laboratory data and the abdominal CT scan, frequently the clinician finds no evidence of alcoholinduced hepatitis or pancreatitis that could be the cause of abdominal pain in patients suffering from alcohol abuse. Upon the suspicion of ischemic colitis, the patient was treated with intravenous hydration, bowel rest, and broad-spectrum antibiotics. After 5 days of treatment, he still had intermittent fever above $39^{\circ} \mathrm{C}$ and severe cramping abdominal pain. On day 7, an abdominal CT scan and colonoscopy were performed repeatedly. Diffuse wall thickening of the terminal ileum to the transverse colon and a large amount of ascites in the pelvic cavity were noted in the CT scan (Fig. 3). There were severe ischemic changes. Mucosal edema and multiple phlegmons were found on the ascending colon with colonoscopy (Fig. 4). On the 8th day after admission, symptoms such as diffuse abdominal distension, abdominal tenderness and rebound tenderness were aggravated; hence, a laparotomy was performed.

When the abdomen was opened, a small amount serous colored
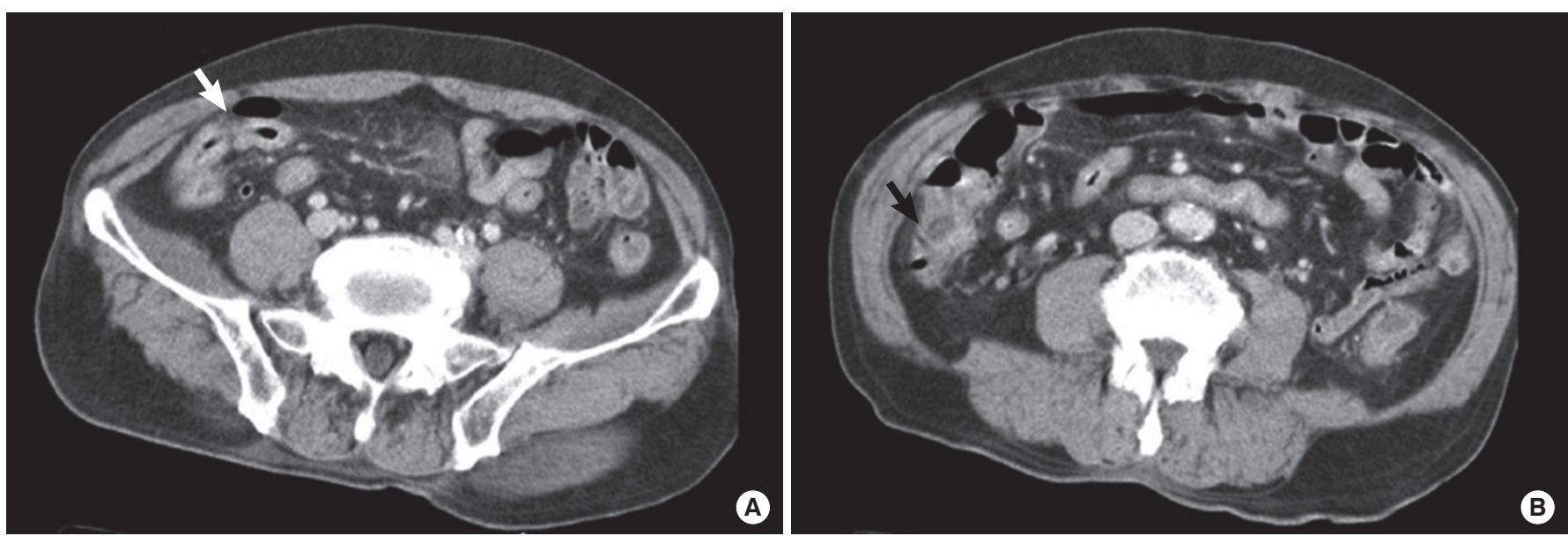

Fig. 2. Abdomen-pelvic computed tomography scan findings on admission day. (A) The white arrow indicates wall thickening of the terminal ileum, and (B) the white arrow indicates wall thickening and inflammatory changes on the cecum and pericolic fat infiltration. 

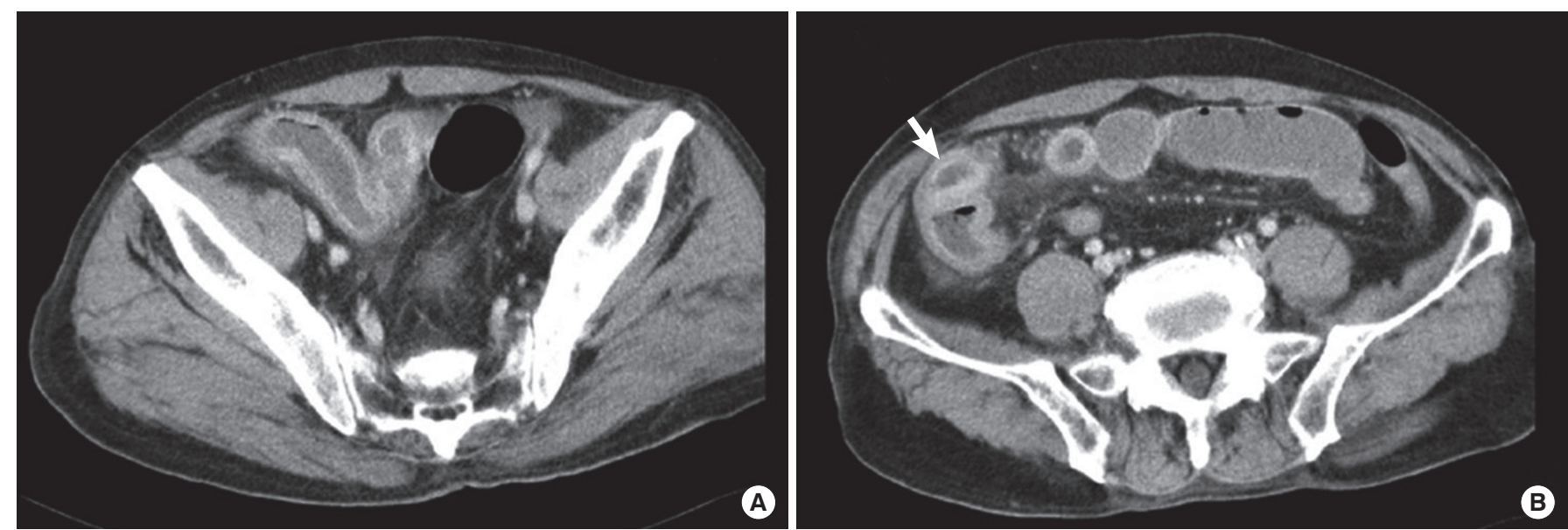

Fig. 3. Abdomen-pelvic computed tomography (CT) scan findings on hospital day 14. Compared to the previous CT scan (A), this abdomenpelvic CT scan (B) shows aggravation of diffuse wall thickening and dilatation of the distal ileum (arrow).
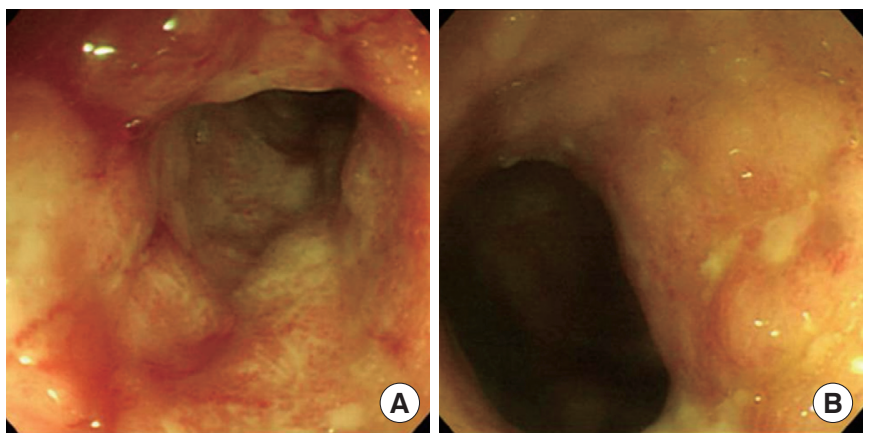

Fig. 4. Colonoscopic finding. There were severe ischemic changes, mucosal edema and multiple phlegmons on the ascending colon. (A) cecum; (B) ascending colon.

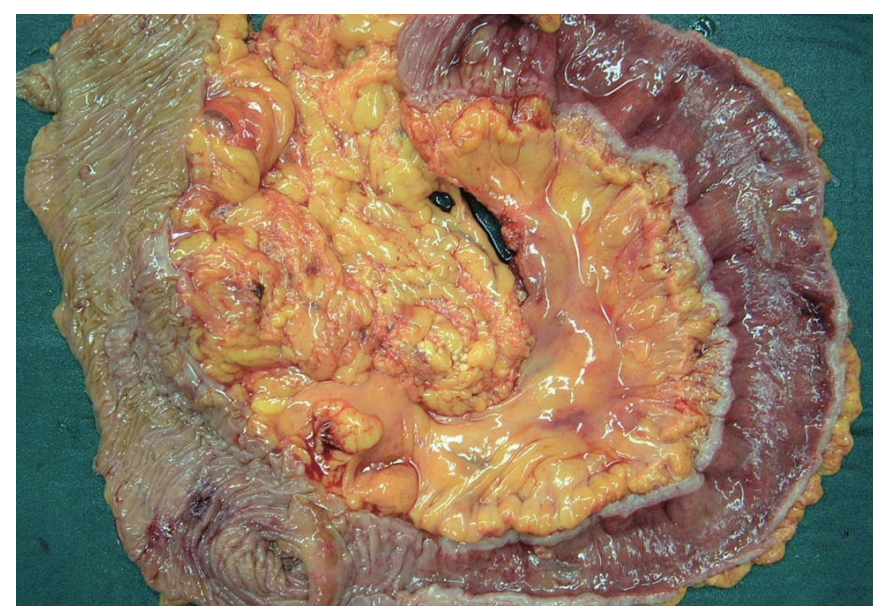

Fig. 5. Macroscopic appearance of the resected specimen. On the section, there is extensive mucosal necrosis, which was associated with inflammation of the mucosa and vascular congestion.

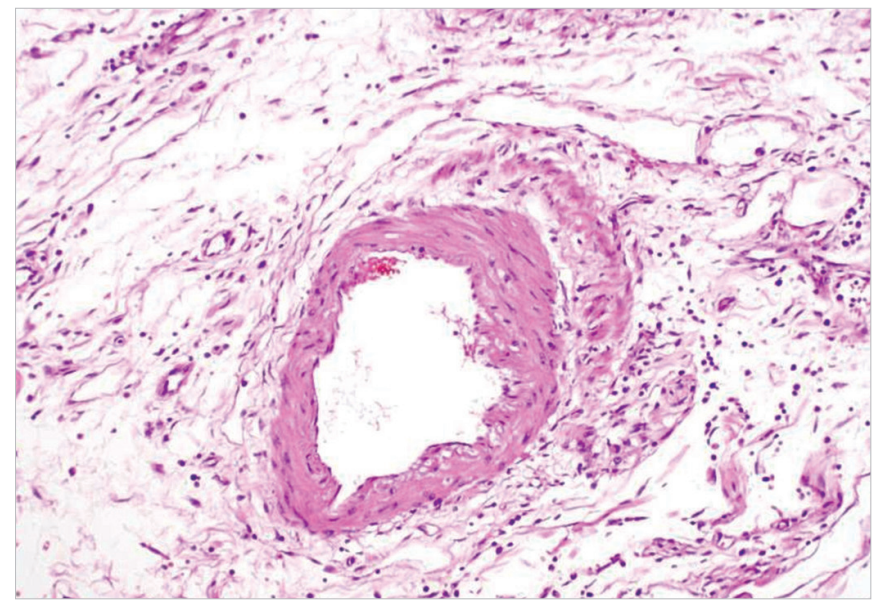

Fig. 6. Microscopic finding. In the terminal ileum, small- to mediumsized arteries in the submucosa show no obvious vasculitis or thrombosis $(\mathrm{H} \& \mathrm{E}, \times 100)$.

ascites was noted in the pelvic cavity. The outer surface of the ileum (from the ileocecal valve to the proximal $30 \mathrm{~cm}$ ) looked dark brown to blackish with severe edema and a weakening of the tissues of the bowel wall was observed. Also, the findings of the cecum and the ascending colon appeared to be similar to those of the affected ileum. The gross appearances of the transverse and the descending colon and the sigmoid colon were almost normal. We performed a right hemicolectomy, including ileum darkened (Fig. 5). Pathologically, a diffuse acute infarction was present in the ileum and cecum, with acute vasoconstrictions of the mesenteric vessels. No atherosclerotic changes or thrombotic occlusions were present on the mesenteric vessels (Fig. 6). A polyp of $0.4 \mathrm{~cm}$ was found on the ascending colon, $13 \mathrm{~cm}$ distal to the ileocecal valve, and was revealed to be a mucosal adenocarcinoma. The postoperative course was uncomplicated, and the patient was discharge 
in good condition on the 15th postoperative day.

\section{DISCUSSION}

NOMI, the dominant non-thrombotic cause of acute mesenteric ischemia, results from low-flow states (cardiogenic shock, sepsis, and hypovolemia), vasoconstriction, or occasionally drugs. NOMI is defined as "intestinal infarction without mesenteric vascular occlusion," and is the most lethal form of AMI, with mortality rates of up to $70 \%[3,4]$. It is a rare condition, but the incidence has been increasing these days. The condition commonly occurs in elderly patients, but a subset of young patients with mesenteric ischemia has been reported in the literature. In this young population, etiologies were reported as hypovolemic shock, vasculitis, and drug use. Medication and addictive drugs, such as digitalis, oral contraceptives, phenobarbital, ergotamine, furosemide and cocaine, have been implicated in the development of ischemic intestinal disease.

For a long time, alcohol has been known to be a precipitating factor of vasodilatation; thus, after alcohol intake, the face becomes red, the blood pressure falls and the blood flow increases in the splanchnic vasculature. Unlike such physiologic changes, pathological binge drinking is known to act as a vasocontricting situation and to be the cause of cardiovascular events. During the past ten years, alcohol consumption has come to be known as a risk factor for developing cerebral vascular diseases, such as stroke. Several studies suggest that ethanol produces prolonged constrictions in cerebral blood vessels, and the vasoconstrictive actions of ethanol are involved in the hypoxic, ischemic and hemorrhagic actions on the brain $[5,6]$. Although several studies have investigated alcohol's effect on the vascular system, there are no reports that alcohol-caused mesenteric vasoconstriction directly causes intestinal ischemia.

The mechanism of alcohol-induced splanchnic vasoconstriction is unclear and is thought to be multifactorial. Several mechanisms want for consideration to explain how alcohol causes bowel ischemia in spite of the difficulty in proving a direct causal relationship between the abuse of alcohol and intestinal ischemia. At first, alcohol may have a direct toxic effect on the gut mucosa and increase bacterial overgrowth in the small intestine $[7,8]$. This persistent mucosal irritation is also likely to impair the susceptibility to abundant normal bacterial floras inside the bowel. Another indirect effect of alcohol on the mucosal microcirculation is an enhanced trans-capillary fluid filtration, increasing the permeability of the mucosa to macromolecules, such as endotoxin and/or other bacterial toxin that cause sepsis [7]. In such a state, the intestinal infection has already started and may result in an irreversible clinical course. Also, alcohol ingestion may have an effect on the blood-clotting system [9]. Alcohol stimulates plasminogen activator inhibitor-1 production and suppresses fibrinolysis. Alcohol intake induces abnormalities in the plasma proteins that are required for blood clotting, leading to the formation of blood clots and the thrombotic occlusion of arterioles. Alcohol may induce mesenteric vasoconstriction directly by suppressing the endothelium-dependent vasorelaxation, leading to reduced blood flow and ischemia [10].

In our case, the patient had no history of any specific infection, had no predisposing factors for systemic vascular problems, and was healthy for his age. We tried conservative treatment under the clinical diagnosis of acute gastroenteritis because there was no evidence of thrombus or constricted vessels in the first series of CT scans, but, in spite of bowel rest and conservative management, the symptoms continued for 15 days (including the treatment period at another hospital) without improvement. There were some differences between the operation findings and the CT findings. During surgery, the ischemic change in the sigmoid colon was not certain. However, the distal ileum to the mid-transverse colon showed diffuse ischemic change, and there were much ascites. Pathological examination of the mesenteric vessels harvested revealed neither thrombosis or embolus within arteries or veins nor evidence of inflammatory bowel disease, such as systemic vasculitis and chronic granulomatous inflammation. Extensive mucosal necrosis, which was associated with inflammation of the mucosa and vascular congestion, was consistent with NOMI. The postoperative course was uneventful, and the patient was discharged without specific complications. It is doubtful that the clinical course of this patient would have been positive if prompt surgery had not been performed.

Recently, with increasing average life span, the elderly population has been increasing rapidly. Many studies on systemic vascular disorders in the elderly have been reported. In the case of emergency situations such as cerebrovascular disease or cardiovascular disease, the best procedure has been established relatively well. However, there are frequent occasions where even doctors cannot diagnose an intestinal infarction when a patient presents with an acute abdomen. The accuracy with which to make this diagnosis is currently unknown. Fortunately, with the improvement of high resolution CT, the accuracy of the diagnosis of an acute abdomen in the elderly is much improved. CT may be helpful in determining the primary cause of bowel ischemia and in allowing direct evaluation of the bowel wall, the adjacent mesentery, and vascular structures. CT is fundamental for early diagnosis and management when evaluating elderly patients with an acute abdomen, and we should have intestinal infarction in mind when evaluating patients with a history of binge drinking.

Drug abuse, including excessive alcohol consumption in particular, has become a major health problem in Korea. This report established that, for successful treatment, mesenteric ischemia should be considered in the differential diagnosis of an acute abdomen in patients with a history of alcohol abuse.

\section{CONFLICT OF INTEREST}

No potential conflict of interest relevant to this article was reported. 


\section{REFERENCES}

1. Gandhi SK, Hanson MM, Vernava AM, Kaminski DL, Longo WE. Ischemic colitis. Dis Colon Rectum 1996;39:88-100.

2. Ullery BS, Boyko AT, Banet GA, Lewis LM. Colonic ischemia: an under-recognized cause of lower gastrointestinal bleeding. J Emerg Med 2004;27:1-5.

3. Clark ET, Gewertz BL. Intermittent ischemia potentiates intestinal reperfusion injury. J Vasc Surg 1991;13:601-6.

4. Yasuhara H. Acute mesenteric ischemia: the challenge of gastroenterology. Surg Today 2005;35:185-95.

5. Yang ZW, Wang J, Zheng T, Altura BT, Altura BM. Ethanol-induced contractions in cerebral arteries: role of tyrosine and mito- gen-activated protein kinases. Stroke 2001;32:249-57.

6. Altura BM, Altura BT. Peripheral vascular actions of ethanol and its interaction with neurohumoral substances. Neurobehav Toxicol Teratol 1983;5:211-20.

7. Bode C, Bode JC. Effect of alcohol consumption on the gut. Best Pract Res Clin Gastroenterol 2003;17:575-92.

8. Beck IT, Dinda PK. Acute exposure of small intestine to ethanol: effects on morphology and function. Dig Dis Sci 1981;26:817-38.

9. Ballard HS. The hematological complications of alcoholism. Alcohol Health Res World 1997;21:42-52.

10. Horie Y, Ishii H. Effect of alcohol on organ microcirculation: its relation to hepatic, pancreatic and gastrointestinal diseases due to alcohol. Nihon Arukoru Yakubutsu Igakkai Zasshi 2001;36:471-85. 